\title{
Risk of recurrence after two children with central nervous system malformations in an area of high incidence
}

\author{
N C NEVIN AND W P JOHNSTON \\ From the Department of Medical Genetics, The Queen's University of Belfast, \\ Institute of Clinical Science, Grosvenor Road, Belfast BT12 6BJ, Northern Ireland
}

SUMMARY This study was undertaken to provide an estimate of the recurrence risk of central nervous system (CNS) malformation in families with two or more affected children. The hospital records and the records of the Genetic Counselling Clinics were examined for instances where a child born between 1962 to 1973 had spina bifida and anencephalus and was documented as having an affected elder sib; such children were taken as index patients. Parents of 76 families with 89 index patients were interviewed. Fifty-one index patients had younger sibs; of 85 younger sibs, 12 had spina bifida and six anencephalus ( 1 in $4 \cdot 7$ ). Little variation was observed in the proportion of subsequent children affected with the type of lesion in the index patient and the affected older sib. Ten index patients with two older affected sibs had 14 subsequent sibs of whom four had a CNS malformation (1 in 3.5). For the purpose of genetic counselling it was suggested that the risk after two and three children with CNS malformations in Northern Ireland was 1 in 5 and 1 in 4, respectively.

In the United Kingdom anencephalus and spina bifida (CNS malformations) show a marked geographical gradient, which steadily increases from the south and east to the north and west; the incidence (per 1000 total births) in south-east England is $2 \cdot 95,{ }^{1}$ in Wales $7 \cdot 6,{ }^{2}$ and in Northern Ireland $8 \cdot 7 .^{3}$ In England, after the birth of an affected child, the risk of a second affected child is 1 in 25 , and after two affected children the risk of recurrence of another affected child is 1 in $8 .{ }^{4}$ It has been suggested that this risk probably varies from one area to another depending on the incidence in the particular population. The incidence of CNS malformations is extremely high in Northern Ireland; in Belfast (1964 to 1968 ), the incidence (per 1000 total births) was $4 \cdot 2$ for anencephalus and 4.5 for spina bifida, ${ }^{3}$ and in Northern Ireland (1974 to 1977) the corresponding figures were $3 \cdot 1$ and $4 \cdot 0 .{ }^{5}$ We report the findings of a study of families designed to give an estimate of the recurrence risk after two affected children.

\section{Material and methods}

The hospital records of the major Northern Ireland maternity units and of the Spina Bifida Clinic, Royal Belfast Hospital for Sick Children were Received for publication 7 April 1979 examined for the years 1962 to 1973 for patients with spina bifida or anencephalus, who had at least one older sib with either of these malformations. The records of the Genetic Counselling Clinic, established in October 1967, were also used to identify similar families. Data on families with anencephalus and spina bifida were available also from a study in Belfast (1964 to 1968). The records were examined at the latest possible date and backwards through the earlier years. An index case was defined as any child with anencephalus or spina bifida or both recorded between 1962 and 1973 who had an affected older sib.

A total of 80 families was identified, but four families could not be traced. The parents of the 76 families were interviewed; there were 89 index patients in these sibships. A full family history, with particular reference to any subsequent sib born up to the last day of 1978 , was documented. The information recorded at the interview was supplemented also from hospital records and from registration of deaths. Details of the sibships are given in the appendix.

Of the 89 index patients, 51 had younger sibs and so provided useful information. There were 85 children born after an index patient. These children were examined personally $(\mathrm{NCN})$ or, if they were 
dead, any congenital abnormality confirmed from hospital records. Thirteen families had two index patients, of which seven had children born after the second index patient.

\section{Results}

The findings among the children born after the 51 index patients are shown in table 1, distinguishing between spina bifida and anencephalus in the index patient and their sibs. Of the 85 subsequent children, proportion of 1 in $4 \cdot 7$. Of 41 subsequent brothers, 10 were affected ( 1 in $4 \cdot 1)$, and of the 44 subsequent sisters, eight were affected (1 in $5 \cdot 5)$. Considering only families in which the index patient was the second born, so that the mother had only affected children, the proportion of subsequent children affected was 3 in 19, that is 1 in 6.33 . This figure is similar to that of the group where the index patient had only one older affected sib: of 65 subsequent children, 13 were affected, a proportion of 1 in 5 .

There was little variation in the proportion of subsequent children with the same type of lesion as the index patient and the affected older sib. When the index patient and the affected older sib each had spina bifida, then of the subsequent 22 children, four had spina bifida, a proportion of 1 in $5 \cdot 5$. Similarly, when the index patient and the older affected sib had anencephalus, of the subsequent 15 children born, three were affected, a proportion of 1 in 5 . Even when the index patient and the affected older sib had dissimilar lesions, the proportion of affected among subsequent born sibs was similar; of the subsequent 28 sibs, six were affected, a proportion of 1 in $4 \cdot 67$.

There were 10 index patients with two older affected sibs. Of the 14 subsequent sibs, four (three with spina bifida and one with anencephalus) were affected, a proportion of 1 in $3 \cdot 5$. One family with two index patients had 11 children, of whom five had spina bifida. The first index patient in this family had three older affected sibs; of the four subsequent sibs one had spina bifida. It so happened that after 12 had spina bifida and six had anencephalus, a

the second index case, who had four affected older sibs, the two subsequent sibs were both normal.

Of the 85 sibs born after the index patient, only one had a congenital abnormality, Down's syndrome, $\stackrel{\vec{S}}{\rightarrow}$ other than a CNS malformation. Of the 163 children $\overline{0}$ born before the index patient two had malformations 흐 other than those of the central nervous system; one $\frac{\bar{\rho}}{\partial}$ had oesophageal atresia, tracheo-oesophageal fistula, $\underset{\mathbb{D}}{\stackrel{2}{2}}$ common atrium, coarctation of the aorta, and hydronephrosis, and the other had talipes equinovarus.

Information was recorded also for cousins of index patients (table 2). Among 620 cousins on the maternal $\overrightarrow{\vec{\omega}}$ side, nine had spina bifida and four anencephalus, a $\stackrel{\vec{\rho}}{\omega}$ proportion of 1 in 48 . Among the 637 paternal first cousins, seven had spina bifida and two had anencephalus, a proportion of 1 in $70 \cdot 8$. Overall among $\vec{v}$ the 1257 cousins, 16 had spina bifida and six had i anencephalus, a proportion of 1 in 57. Thus, the $\stackrel{\infty}{v}$ incidence of CNS malformations among cousins is 우 $2 \cdot 5$-fold that of the general population. In addition $\rightarrow$ to the CNS malformations among cousins, one of $\frac{D}{2}$ mother's brother's daughters had hydrocephalus, and one of father's sister's daughters had spina bifida $\vec{\bullet}$ occulta. Eleven cousins had other congenital mal- ${ }^{\circ}$ formations: five had congenital heart defects, one had duodenal atresia, one had talipes equinovarus, one had mental retardation, one had Down's syndrome, and two had epilepsy.

The findings among the cousins in the families with two index patients is shown in table 3. Among

TABLE 2 Malformations among cousins of index patients

\begin{tabular}{llll}
\hline Relation & Total & Spina bifida & Anencephalus \\
\hline Maternal & & & \\
$\quad$ Brother's sons & 148 & 1 & 1 \\
Brother's daughters & 144 & 2 & 1 \\
Sister's sons & 164 & 3 & 0 \\
Sister's daughters & 164 & 3 & 2 \\
Total & 620 & 9 & 4 \\
Paternal & 158 & 0 & 0 \\
$\quad$ Brother's sons & 155 & 2 & 0 \\
Brother's daughters & 167 & 2 & 1 \\
$\quad$ Sister's sons & 157 & 3 & 1 \\
Sister's daughters & 637 & 7 & 2 \\
Total & & & \\
\hline
\end{tabular}

TABLE 1 Children born after index patient




TABLE 3 Malformations among cousins of families with two index patients

\begin{tabular}{lrll}
\hline Relation & Total & Spina bifida & Anencephalus \\
\hline Maternal & & & \\
$\quad$ Brother's sons & 38 & 1 & 1 \\
Brother's daughters & 36 & 1 & 0 \\
Sister's sons & 41 & 0 & 0 \\
Sister's daughters & 44 & 1 & 0 \\
Total & 159 & 3 & 1 \\
Paternal & & & \\
$\quad$ Brother's sons & 48 & 0 & 0 \\
Brother's daughters & 36 & 1 & 0 \\
$\quad$ Sister's sons & 54 & 1 & 0 \\
Sister's daughters & 40 & 3 & 0 \\
Total & 178 & 5 & 0 \\
\hline
\end{tabular}

TABLE 4 Malformations among uncles and aunts of index patients

\begin{tabular}{llll}
\hline Relation & Total & Spina bifida & Anencephalus \\
\hline Maternal & & & \\
$\quad$ Uncles & 187 & 2 & 0 \\
$\quad$ Aunts & 155 & 2 & 0 \\
Total & 342 & 4 & 0 \\
Paternal & & & \\
$\quad$ Uncles & 177 & 0 & 0 \\
Aunts & 156 & 2 & 0 \\
Total & 333 & 2 & 0 \\
\hline
\end{tabular}

159 maternal cousins, three had spina bifida and one anencephalus, a proportion of 1 in 39.8 . Among 178 paternal cousins, five had spina bifida, a proportion of 35.6 . In all of 337 cousins, nine had CNS malformations, a proportion of 1 in $37 \cdot 4$, which is $3 \cdot 8$ times the general population incidence.

Data were collected on CNS malformations among uncles and aunts of the index patients, but these were far from complete as it was difficult to trace hospital records to confirm diagnoses, and death certificates often only recorded stillbirth or neonatal death without mention of congenital abnormalities. Of 675 uncles and aunts, six had spina bifida, a proportion of 1 in $112 \cdot 5$, which is similar to that for the general population and is clearly an underestimate.

\section{Discussion}

The risk of recurrence of anencephalus or spina bifida after the birth of two affected children is considered to be much higher than after one affected child. In England, the risk of recurrence after the birth of a single affected child is about 1 in 25.4 Lorber ${ }^{6}$ reported 13 families who had two children with spina bifida; of 67 subsequent births, 39 (58\%) were affected. The author, however, was careful to point out that these families were highly selected, as families with a third or fourth affected child had been referred for treatment in a period when surgical treatment had been more generally recognised.
Smithells et $a l^{7}$ also found an unusually high recurrence rate after a second infant with anencephalus or spina bifida. Again, their study was biased towards sibships with two affected infants as their registry was established after the birth of the second. They found that half of the children born after the birth of the second infant had a CNS malformation. In Glasgow, Richards et $a l^{8}$ found the overall incidence of CNS malformations to be $4.9 \%$ after two affected children, a lower rate than found in other studies.

In a particularly well-designed investigation, Carter and Roberts 4 interviewed, from various centres in England and Wales, 47 families which had children born after the birth of two affected children. Of 69 subsequent children, four had spina bifida and four had anencephalus, a proportion of 1 in $8 \cdot 6$. For the purpose of genetic counselling they suggested that the risk in England after two children with neural tube defects is 1 in 10.

Carter and Roberts ${ }^{4}$ suggested that the risk of recurrence might be expected to vary from one area to another, depending upon the incidence of these malformations in the particular population. As Northern Ireland has a very high incidence of neural tube defects, it was considered appropriate to investigate the risk of recurrence after two affected children, so that comparison could be made with the study of Carter and Roberts. ${ }^{4}$ Of 85 children born after two affected children, 12 had spina bifida and six had anencephalus, an overall recurrence risk of 1 in $4 \cdot 7$, which is almost twice that found by Carter and Roberts. 4

In the study of Carter and Roberts, ${ }^{4}$ eight children were born after three affected children, and it so happened that all were normal. In our study there were 10 index patients with two older affected sibs. There was a total of 14 children born after the index patient, of whom four were affected (a proportion of 1 in $3 \cdot 5$ ). It is apparent that with each successive affected child, the risk of recurrence of neural tube defects rises steadily. For the purpose of genetic counselling in Northern Ireland, it would seem reasonable to advise a recurrence risk of 1 in 5 and 1 in 4 , after the birth of two and three affected children, respectively.

We wish to thank all our colleagues who allowed us access to their records and all the families for their co-operation. We are also indebted to Professor C O Carter, MRC Clinical Genetics Unit, London, for his helpful comments on this paper. This research project was supported by the Belfast Branch, and the Lurgan and Portadown Branch, of the Northern Ireland Association for Spina Bifida and Hydrocephalus. 


\section{References}

1 Carter CO, Evans K. Spina bifida and anencephalus in Greater London. J Med Genet 1973;10:209-34.

2 Laurence KM, Carter CO, David PA. Major central nervous system malformations in South Wales. Br J Prev Soc Med 1968;22:147-212.

${ }^{3}$ Elwood JH, Nevin NC. Factors associated with anencephalus and spina bifida in Belfast. Br J Prev Soc Med $1973 ; 27: 73-80$.

4 Carter CO, Roberts JAF. The risk of recurrence after two children with central nervous system malformations. Lancet 1967;1:306-8.

5 Nevin NC, McDonald JR, Walby AL. A comparison of neural tube defects identified by two independent routine recording systems for congenital malformations in Northern Ireland. Int J Epidemiol 1978;7:319-21.
${ }^{6}$ Lorber J. The family history of spina bifida cystica. $\stackrel{\mathbb{Q}}{7}$ Pediatrics 1965;35:589-95.

7 Smithells RW, D'Arcy EE, McAllister EF. Outcome of '? pregnancies before and after birth of infants with nervous $\vec{\overrightarrow{ }}$ system malformations. Dev Med Child Neurol (Suppl)$1968 ; 15: 6-10$.

8 Richards IDG, McIntosh HT, Sweenie S. A genetic study $\frac{\bar{O}}{\bar{c}}$ of anencephaly and spina bifida in Glasgow. Dev Med $\vec{\Phi}$ Child Neurol 1972;14:626-39.

Requests for reprints to Professor N C Nevin, $\overrightarrow{0}$ Department of Medical Genetics, Queen's University of Belfast, Institute of Clinical Science, Grosvenor $\vec{\omega}$ Road, Belfast BT12 6BJ, Northern Ireland. 
APPENDIX Index patients and sibs

$M$, male; $F$, female; $m$, miscarriage; sb, stillbirth ; ${ }^{*}$ index patient; [ ], twins; $(A)$, anencephalus; $(S)$, spina bifida; $(H)$, hydrocephalus.

\begin{tabular}{|c|c|c|c|c|}
\hline \multirow[t]{2}{*}{ Serial No } & \multirow[t]{2}{*}{ Sibship } & \multicolumn{2}{|c|}{ Date of birth } & \\
\hline & & Mother & Father & \\
\hline \multicolumn{4}{|c|}{$\begin{array}{l}\text { A. SPINA BIFIDA (INDEX PATIENT): SPINA BIFIDA (OLDER SIB) } \\
\text { Two child families }\end{array}$} & \\
\hline 010 & $\mathrm{M}(\mathrm{S}) 3 / 71 \mathrm{sb} ;{ }^{*} \mathrm{M}(\mathrm{S}) 1 / 72 ; \mathrm{ml} / 73 ; \mathrm{m} 4 / 75, \mathrm{ml} / 76$ & 1949 & 1947 & Q \\
\hline 011 & $\mathbf{M}(\mathbf{S}) 3 / 71 ; * \mathbf{M}(\mathbf{S}) 11 / 72$ & 1954 & 1952 & के \\
\hline 021 & $\mathbf{M}(\mathbf{S}) 5 / 70 ; \mathbf{M}(\mathbf{S}) 9 / 71$ & 1947 & 1944 & - \\
\hline 083 & $\mathrm{~F}(\mathrm{~S}) 7 / 61 ;{ }^{*} \mathrm{M}(\mathrm{S}) 4 / 62 \mathrm{sb} ; \mathrm{m5} / 66 ; \mathrm{m5} / 67$ & 1938 & 1933 & $c$ \\
\hline \multicolumn{4}{|c|}{ Three child families } & \\
\hline 001 & $F 8 / 69 ; F(S) 8 / 70 ; * F(S) 4 / 73$ & 1946 & 1942 & $\omega$ \\
\hline 012 & $\mathrm{~F}(\mathrm{~S}) 7 / 67 \mathrm{sb} ; \mathrm{M} 3 / 69 ; * \mathbf{F}(\mathrm{S}) 3 / 72$ & 1942 & 1943 & g \\
\hline 022 & $\mathbf{M}(\mathbf{S}) 7 / 69 ; * \mathbf{M}(\mathbf{S}) 2 / 71 ; \mathbf{F} 2 / 72$ & 1947 & 1947 & ב \\
\hline & M10/66;m- $/ 67 ; \mathrm{m}-/ 68 ; \mathrm{F}(\mathrm{S}) 7 / 69 ; \mathrm{m}-/ 70 ;{ }^{*} \mathrm{~F}(\mathrm{~S}) 7 / 71$ & 1947 & 1944 & (3) \\
\hline 044 & $\mathrm{M}(\mathrm{S}) 12 / 64 ; \mathrm{m} 5 / 65 ; \mathrm{M} 6 / 66 ; * \mathrm{M}(\mathrm{S}) 2 / 69$ & 1943 & 1940 & \\
\hline 051 & $\mathbf{M}(\mathbf{S}) 4 / 66 ; \mathrm{m}-167 ; * \mathbf{M}(\mathbf{S}) 2 / 68 ; \mathrm{F} 5 / 72 ; \mathrm{m} 3 / 73$ & 1940 & 1942 & $=$ \\
\hline & $\mathrm{F}(\mathrm{S}) 1 / 61 ; \mathrm{F} 9 / 64 ; \mathrm{M}(\mathrm{S}) 4 / 66 \mathrm{sb} ; \mathrm{m6} / 72$ & 1941 & 1940 & $\dot{n}$ \\
\hline & $\mathrm{M}(\mathrm{S}) 1 / 59 ; \mathrm{F} 5 / 61 ; * \mathrm{~F}(\mathrm{~S}) 7 / 66$ & 1936 & 1939 & $\alpha$ \\
\hline \multicolumn{4}{|c|}{ Four child families } & \\
\hline 023 & $\mathrm{M}(\mathrm{S}) 6 / 69 ; \mathrm{ml} / 70 ;{ }^{*} \mathrm{~F}(\mathrm{~S}) 1 / 71 ; \mathrm{M} 11 / 73 ; \mathrm{F} 9 / 78$ & 1950 & 1946 & 으 \\
\hline 076 & $\mathrm{M} 11 / 54 ; \mathrm{F} 7 / 56 ; \mathrm{F}(\mathrm{S}) 9 / 61 ;{ }^{*} \mathrm{~F}(\mathrm{~S}) 1 / 64 ; \mathrm{m5} / 67$ & 1934 & 1931 & \\
\hline \multicolumn{5}{|c|}{ Five child families } \\
\hline 002 & $\mathrm{~F} 3 / 68 ; \mathrm{F} 6 / 69 ; \mathrm{m} 9 / 71 ; \mathrm{F}(\mathrm{S}) 1 / 73 ; * \mathrm{~F}(\mathrm{~S}) 11 / 73 \mathrm{sb} ; \mathrm{F} 12 / 74$ & 1938 & 1928 & 1 \\
\hline & $\mathrm{m} 3 / 63 ; \mathrm{M}(\mathrm{S}) 2 / 64 ; \mathrm{M} 2 / 65 ; \mathrm{M} 6 / 66 ; \mathrm{M} 5 / 67 ; \mathrm{M}(\mathrm{S}) 6 / 69 ; \mathrm{m} 11 / 71$ & 1941 & 1935 & 을 \\
\hline 056 & $\mathrm{~F}(\mathrm{~S}) 1 / 61 ; \mathrm{M} 3 / 62 ; \mathrm{M} 2 / 65 ;{ }^{*} \mathrm{~F}(\mathrm{~S}) 5 / 67 ; \mathrm{F} 2 / 69$ & 1935 & 1933 & \\
\hline 062 & $\mathrm{M}(\mathrm{S}) 11 / 63 ; \mathrm{m} 12 / 63 ; \mathrm{M} 1 / 65 ; * \mathrm{~F}(\mathrm{~S}) 3 / 66 ; \mathrm{M} 2 / 67 ; \mathrm{F} 1 / 70$ & 1942 & 1943 & $\bar{c}$ \\
\hline & $\mathrm{F} 1 / 62 ; \mathrm{M}(\mathrm{S}) 3 / 63 ; \mathrm{M} 7 / 64 ; * \mathrm{~F}(\mathrm{~S}) 11 / 65 ; \mathrm{F} 4 / 69$ & 1940 & 1940 & $\propto$ \\
\hline 073 & $\mathrm{M} 9 / 63 ; \mathrm{M}(\mathrm{S}) 4 / 64 ; * \mathrm{~F}(\mathrm{~S}) 12 / 65 ; \mathrm{m}-/ 67 ; \mathrm{m}-/ 69 ; \mathrm{M} 5 / 70 ; \mathrm{M} 3 / 72$ & 1941 & 1941 & 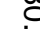 \\
\hline \multicolumn{4}{|c|}{ Six child families } & c \\
\hline 025 & M3/66;F5/67;F9/69;M(S)11/70;*M(S)12/71;M5/73 & 1945 & 1943 & c \\
\hline & M11/61;m- /62;F10/64;M(S) $12 / 65 ; M 5 / 66 ; M 5 / 68 ; * \mathrm{~F}(\mathrm{~S}) 3 / 71$ & 1942 & 1942 & \\
\hline 027 & $\mathrm{M} 12 / 61 ; \mathrm{M} 11 / 64 ;[\mathrm{M}(\mathrm{S}) 10 / 67 ; \mathrm{F}(\mathrm{S}) 10 / 67] ; \mathrm{F} 8 / 70 ;{ }^{*} \mathrm{M}(\mathrm{S}) 2 / 71$ & 1942 & 1940 & 己 \\
\hline & $\mathrm{M} 8 / 64 ; \mathrm{F}(\mathrm{S}) 12 / 65 ; \mathrm{M} 3 / 66 ; * \mathrm{M}(\mathrm{S}) 9 / 68 ; \mathrm{F} 5 / 69 ; \mathrm{M} 12 / 70 \mathrm{sb} ; \mathrm{m10} / 71 ; \mathrm{F} 2 / 74 ; \mathrm{m} 8 / 75$ & 1944 & 1943 & 0 \\
\hline & $\mathrm{M} 4 / 56 ; \mathrm{M} 11 / 57 ; \mathrm{F}(\mathrm{S}) 2 / 60 ; \mathrm{M} 4 / 63 ; \mathrm{F}(\mathrm{S}) 6 / 65 ; \mathrm{M}(\mathrm{S}) 8 / 66$ No 064 & 1936 & 1939 & 象 \\
\hline 085 & $\mathrm{M}(\mathrm{S}) 11 / 52 ; \mathrm{m}-/ 53 ; \mathrm{M}-/ 54 ; \mathrm{F} 4 / 55 ; \mathrm{F} 5 / 56 ; \mathrm{M}-/ 59 ;{ }^{*} \mathrm{M}(\mathrm{S}) 1 / 62$ & 1935 & 1935 & (D) \\
\hline \multicolumn{4}{|c|}{ Seven + child families } & \\
\hline & m10/63;M(S)12/64;M1/66;m6/66;F8/67;F7/68;*M(S)4/70;M(S)8/71 No 028;F(S)1/74 & 1940 & 1932 & 윽 \\
\hline & $\mathrm{m5} / 56 ; \mathrm{M} 3 / 57 ; \mathrm{m12} / 57 ; \mathrm{F}(\mathrm{S}) 1 / 59 ; \mathrm{M} 6 / 60 ; \mathrm{m12} / 60 ; \mathrm{M} 7 / 62 ; \mathrm{m5} / 63 ; * \mathrm{M}(\mathrm{S}) 9 / 64 ; \mathrm{m}-/ 65 ; \mathrm{F} 7 / 66 ; \mathrm{F} 11 / 69$ & 1935 & 1929 & 3 \\
\hline 086 & F10/54;F12/55;M2/56sb;M1/58;F(S)4/59;M8/60;m4/61;*F(S)2/62;M(S)7/64 No 078;F10/69 & 1935 & 1933 & \\
\hline \multicolumn{4}{|c|}{$\begin{array}{l}\text { B. ANENCEPHALUS (INDEX PATIENT): ANENCEPHALUS (OLDER SIB) } \\
\text { Two child families }\end{array}$} & $\bar{c}$ \\
\hline & $\mathrm{M}(\mathrm{A}) 5 / 70 \mathrm{sb} ; \mathrm{m} 12 / 72 ; * \mathrm{~F}(\mathrm{~A}) 11 / 73 \mathrm{sb}$ & 1936 & 1938 & ב- \\
\hline 004 & $\mathrm{~m} 5 / 71 ; \mathrm{F}(\mathrm{A}) 2 / 72 \mathrm{sb} ;{ }^{*} \mathrm{~F}(\mathrm{~A}) 11 / 73 \mathrm{sb}$ & 1945 & 1946 & قُ \\
\hline & $\mathrm{m} 5 / 68 ; \mathrm{F}(\mathrm{A}) 6 / 69 \mathrm{sb} ; * \mathrm{~F}(\mathrm{~A}) 7 / 71 \mathrm{sb}$ & 1946 & 1945 & (2. \\
\hline 087 & $M(A) 7 / 59 \mathrm{sb} ; \mathrm{m} 4 / 60 ; * \mathrm{~F}(\mathrm{~A}) 12 / 68 \mathrm{sb}$ & 1939 & 1935 & 0 \\
\hline \multicolumn{4}{|c|}{ Three child families } & \\
\hline & $\mathrm{F}(\mathrm{A}) 2 / 72 \mathrm{sb} ;{ }^{*} \mathrm{~F}(\mathrm{~A}) 1 / 73 \mathrm{sb} ; \mathrm{M} 2 / 74$ & 1950 & 1953 & อి \\
\hline & F(A) $4 / 72 \mathrm{sb} ; * \mathbf{F}(\mathrm{A}) 3 / 73 \mathrm{sb} ; \mathrm{M1} / 75$ & 1950 & 1947 & 국 \\
\hline 014 & $\mathrm{~F}(\mathrm{~A}) 2 / 71 ; \mathrm{m}-/ 71 ; * \mathrm{M}(\mathrm{A}) 11 / 72 \mathrm{sb} ; \mathrm{M}(\mathrm{A}) 11 / 73$ No 007 & 1945 & 1947 & \\
\hline 075 & $\mathrm{~F}(\mathrm{~A}) 10 / 58 \mathrm{sb} ; \mathrm{M} 3 / 62 ;{ }^{*} \mathrm{M}(\mathrm{A}) 8 / 65 \mathrm{sb} ; \mathrm{m}-/ 70$ & 1939 & 1932 & 윽 \\
\hline \multicolumn{4}{|c|}{ Four child families } & \\
\hline 030 & $\mathrm{~m}-/ 69 ; \mathrm{F}(\mathrm{A}) 6 / 70 \mathrm{sb} ; * \mathrm{~F}(\mathrm{~A}) 10 / 71 \mathrm{sb} ; \mathrm{M} 8 / 74 ; \mathrm{F} 10 / 76$ & 1945 & 1945 & ర \\
\hline 046 & $\mathrm{~F}(\mathrm{~A}) 8 / 66 \mathrm{sb} ; \mathrm{m11} 166 ; \mathrm{M} 9 / 67 ; * \mathrm{~F}(\mathrm{~A}) 12 / 69 ; \mathrm{F} 3 / 72$ & 1946 & 1947 & 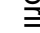 \\
\hline 053 & $\mathrm{~F}(\mathrm{~A}) 10 / 64 \mathrm{sb} ; \mathrm{F} 10 / 65 ; * \mathrm{M}(\mathrm{A}) 2 / 68 \mathrm{sb} ; \mathrm{F} 6 / 69$ & 1939 & 1938 & \\
\hline 054 & F(A) $10 / 65 \mathrm{sb} ; \mathrm{F} 9 / 66 ; \mathrm{m}-/ 67 ; * \mathrm{M}(\mathrm{A}) 7 / 68 \mathrm{sb} ; \mathrm{M} 1 / 77$ & 1945 & 1946 & o \\
\hline \multicolumn{4}{|c|}{ Five child families } & \\
\hline 032 & $\mathrm{M}(\mathrm{A}) 1 / 70 \mathrm{sb} ;{ }^{*} \mathrm{M}(\mathrm{A}) 5 / 71 \mathrm{sb} ; \mathrm{m} 9 / 71 ; \mathrm{F} 1 / 73 ; \mathrm{F}(\mathrm{S}) 1 / 74 \mathrm{sb} ; \mathrm{m} 1 / 76 ; \mathrm{M} 11 / 76$ & 1943 & 1945 & \\
\hline & $F(A) 8 / 66 \mathrm{sb} ; \mathrm{F} 5 / 68 ; * \mathrm{~F}(\mathrm{~A}) 6 / 69 ; \mathrm{F}(\mathrm{A}) 11 / 71 \mathrm{sb}$ No $031 ; \mathrm{M} 10 / 78$ & 1941 & 1940 & \\
\hline \multicolumn{4}{|c|}{ Seven + child families } & \\
\hline 033 & M11/62;m6/64;M4/67;F(A)11/69sb;*F(A)3/71sb;M1/73;F8/74 & 1938 & 1937 & 울 \\
\hline $\begin{array}{l}\text { C. ANENCEP } \\
\text { Two child } f\end{array}$ & $\begin{array}{l}\text { ALUS (INDEX PATIENT): SPINA BIFIDA (OLDER SIB) } \\
\text { ailies }\end{array}$ & & & @ \\
\hline 055 & F(S)9/67;*M(A)8/68 & 1935 & 1938 & \\
\hline Three child & amilies & & & \\
\hline $\begin{array}{l}015 \\
068\end{array}$ & $\begin{array}{l}\operatorname{m12/68;M12/69;F(S)12/70;*F(A)4/72sb~} \\
M(S) 9 / 65 ; * F(A) 8 / 66 s b ; F 2 / 70\end{array}$ & $\begin{array}{l}1949 \\
1944\end{array}$ & $\begin{array}{l}1948 \\
1946\end{array}$ & 2 \\
\hline Four child & milies & & & \\
\hline 034 & $\mathrm{M} 9 / 66 ; \mathrm{m}-/ 67 ; \mathrm{F} 12 / 67 ; \mathrm{M}(\mathrm{S}) 3 / 70 \mathrm{sb} ;{ }^{*} \mathrm{M}(\mathrm{A}) 6 / 71 \mathrm{sb}$ & 1939 & 1942 & \\
\hline & $\mathrm{F}(\mathrm{S}) 7 / 67 ; \mathrm{M} 12 / 68 ;{ }^{*} \mathrm{M}(\mathrm{A}) 4 / 71 \mathrm{sb} ; \mathrm{M} 10 / 72$ & 1934 & 1939 & \\
\hline 036 & $\mathrm{M}(\mathrm{S}) 5 / 69 ; \mathrm{m12} / 69 ; \mathrm{F}(\mathrm{A}) 12 / 71 ; \mathrm{M} 4 / 74 ; \mathrm{M} 5 / 77$ & 1949 & 1950 & \\
\hline & $\mathrm{M} 6 / 64 ; \mathrm{M}(\mathrm{S}) 4 / 67 ; \mathrm{M} 12 / 68 ; * \mathrm{~F}(\mathrm{~A}) 4 / 70 \mathrm{sb}$ & 1937 & 1938 & \\
\hline
\end{tabular}


APPENDIX continued

\begin{tabular}{|c|c|c|c|}
\hline \multirow[t]{2}{*}{ Serial No } & \multirow[t]{2}{*}{ Sibship } & \multicolumn{2}{|c|}{ Date of birth } \\
\hline & & Mother & Father \\
\hline \multicolumn{4}{|c|}{$\begin{array}{l}\text { D. SPINA BIFIDA (INDEX PATIENT): ANENCEPHALUS (OLDER SIB) } \\
\text { Two child families }\end{array}$} \\
\hline 008 & $\begin{array}{l}\text { F(A) } 9 / 71 ; * F(S) 10 / 73 \\
M(A) 7 / 67 ; * F(S) 6 / 70 \text { sb }\end{array}$ & $\begin{array}{l}1950 \\
1939\end{array}$ & $\begin{array}{l}1946 \\
1945\end{array}$ \\
\hline \multicolumn{4}{|c|}{ Three child families } \\
\hline & F(A) $5 / 69 ; \mathrm{m}-/ 70 ;{ }^{*} \mathrm{~F}(\mathrm{~S}) 4 / 72 \mathrm{sb} ; \mathrm{M} 3 / 75$ & 1946 & 1948 \\
\hline 017 & F6 $/ 67 ; F(A) 1 / 71$ sb; $* F(S) 2 / 72$ & 1936 & 1940 \\
\hline & $\mathrm{M}(\mathrm{A}) 4 / 69 \mathrm{sb} ;{ }^{* \mathrm{~F}}(\mathrm{~S}) 6 / 71 ; \mathrm{M} 3 / 73$ & 1938 & 1943 \\
\hline & $\mathrm{F}(\mathrm{A}) 6 / 66 \mathrm{sb} ; \mathrm{F} 3 / 67 ;{ }^{*} \mathrm{M}(\mathrm{S}) 11 / 69$ & 1936 & 1936 \\
\hline \multicolumn{4}{|c|}{ Four child families } \\
\hline 018 & F8/71;F(A)11/72sb;*F(S)12/73;M4/75 & 1935 & 1934 \\
\hline & $\mathrm{F} 2 / 71 ; \mathrm{F}(\mathrm{A}) 1 / 72 \mathrm{sb} ;{ }^{*} \mathrm{M}(\mathrm{S}) 4 / 73 ; \mathrm{M} 3 / 74$ & 1950 & 1949 \\
\hline 049 & F6/63;M(A)9/64sb;M9/65;*M(S)5/69 & 1932 & 1935 \\
\hline 081 & $\mathrm{~F}(\mathrm{~A}) 9 / 61 \mathrm{sb} ; \mathrm{M} 8 / 62 ; * \mathbf{M}(\mathrm{S}) 2 / 64 \mathrm{sb} ; \mathrm{m} 7 / 65 ; \mathrm{M}(\mathrm{A}) 4 / 67$ No 058 & 1939 & 1934 \\
\hline & $\mathrm{m} 1 / 62 ; \mathrm{M}(\mathrm{A}) 10 / 62 \mathrm{sb} ; * \mathrm{M}(\mathrm{S}) 10 / 64 ; \mathrm{F}(\mathrm{S}) 10 / 66$ No $063 ; \mathrm{m} 12 / 67 ; \mathrm{m8} / 68 ; \mathrm{F} 2 / 70$ & 1938 & 1935 \\
\hline \multicolumn{4}{|c|}{ Five child families } \\
\hline & $\mathrm{m}-/ 62 ; \mathrm{M}(\mathrm{A}) 10 / 63 \mathrm{sb} ; \mathrm{M} 11 / 64 ; * \mathrm{M}(\mathrm{S}) 4 / 66 ; \mathrm{F} 7 / 68 ; \mathrm{F}(\mathrm{S}) 11 / 72$ No 019 & 1939 & 1939 \\
\hline & $\mathrm{M} 2 / 63 ; \mathrm{F}(\mathrm{A}) 11 / 64 \mathrm{sb} ; \mathbf{M}(\mathrm{S}) 1 / 66 ; \mathrm{F} 7 / 68 ; \mathrm{F} 1 / 70$ & 1939 & 1937 \\
\hline \multicolumn{4}{|c|}{ Six child families } \\
\hline & $\mathrm{F} 4 / 70 ; \mathrm{F}(\mathrm{A}) 2 / 71 \mathrm{sb} ; * \mathrm{M}(\mathrm{S}) 12 / 72 ; \mathrm{M} 1 / 73 ; \mathrm{m}-/ 73 ; \mathrm{F} 2 / 74 ; \mathrm{F} 8 / 75$ & 1949 & 1949 \\
\hline & $\mathrm{M} 9 / 64 ; \mathrm{F}(\mathrm{A}) 1 / 66 ;{ }^{*} \mathrm{M}(\mathrm{S}) 7 / 67 ; \mathrm{M} 3 / 69 ; \mathrm{M} 6 / 71 ; \mathrm{M} 4 / 74$ & 1934 & 1936 \\
\hline \multicolumn{4}{|c|}{ Seven + child families } \\
\hline & $\mathrm{M}(\mathrm{A}) 9 / 63 \mathrm{sb} ; \mathrm{F} 2 / 65 ; \mathrm{F} 5 / 67 ;{ }^{*} \mathrm{M}(\mathrm{S}) 9 / 70 ; \mathrm{F}(\mathrm{S}) 2 / 73$ No $009 ; \mathrm{F} 2 / 75 ; \mathrm{M} 10 / 77$ & 1936 & 1936 \\
\hline & $\mathrm{M} 2 / 64 ; \mathrm{M}(\mathrm{A}) 8 / 65 \mathrm{sb} ;{ }^{*} \mathrm{M}(\mathrm{S}) 9 / 66 ;[\mathrm{M}(\mathrm{A}) 5 / 69$ No $050 ; \mathrm{M} 5 / 69] ; \mathrm{F} 11 / 70 ; \mathrm{M}(\mathrm{A}) 5 / 76$ & 1941 & 1940 \\
\hline \multicolumn{4}{|c|}{$\begin{array}{l}\text { E. ANENCEPHALUS (INDEX PATIENT): TWO AFFECTED OLDER SIBS } \\
\text { Three child families }\end{array}$} \\
\hline & $\mathrm{F}(\mathrm{A}) 2 / 71 ; \mathrm{m}-/ 71 ; \mathrm{M}(\mathrm{A}) 11 / 72 \mathrm{sb}$ No $014 ; \mathrm{*}(\mathrm{A}) 11 / 73$ & 1945 & 1947 \\
\hline \multicolumn{4}{|c|}{ Four child families } \\
\hline 058 & $\mathrm{~F}(\mathrm{~A}) 9 / 61 \mathrm{sb} ; \mathrm{M} 8 / 62 ; \mathrm{M}(\mathrm{S}) 2 / 64 \mathrm{sb}$ No $081 ; \mathrm{m} 7 / 65 ; \mathrm{M}(\mathrm{A}) 4 / 67$ & 1939 & 1934 \\
\hline \multicolumn{4}{|c|}{ Five child families } \\
\hline 031 & F(A)8/66sb;F5/68;F(A)6/69 No 047;*F(A)11/71sb;M10/78 & 1941 & 1940 \\
\hline \multicolumn{4}{|c|}{ Seven + child families } \\
\hline 050 & M2/64;M(A)8/65sb;M(S)9/66 No 071;[*M(A)5/69;M5/69];F11/70;M(A)5/76 & 1941 & 1940 \\
\hline \multicolumn{4}{|c|}{$\begin{array}{l}\text { F. SPINA BIFIDA (INDEX PATIENT): TWO AFFECTED OLDER SIBS } \\
\text { Three child families }\end{array}$} \\
\hline 080 & $\mathrm{~F}(\mathrm{~S}) 10 / 48 ; \mathrm{m}-/ 49 ; \mathrm{M}(\mathrm{A})-/ 50 ; \mathrm{F}(\mathrm{S}) 3 / 63$ & 1922 & 1921 \\
\hline \multicolumn{4}{|c|}{ Four child families } \\
\hline & $\mathrm{m} 1 / 62 ; \mathrm{M}(\mathrm{A}) 10 / 62 \mathrm{sb} ; \mathrm{M}(\mathrm{S}) 10 / 64$ No $084 ;{ }^{*} \mathrm{~F}(\mathrm{~S}) 10 / 66 ; \mathrm{m} 12 / 67 ; \mathrm{m} 8 / 68 ; \mathrm{F} 2 / 70$ & 1938 & 1935 \\
\hline & $\mathrm{m}-/ 58 ; \mathrm{M}(\mathrm{S}) 9 / 60 ; \mathrm{F}(\mathrm{S}) 10 / 61 ; \mathrm{m}-/ 63 ; \mathrm{F}(\mathrm{S}) 12 / 64 ; \mathrm{F}(\mathrm{S}) 4 / 71$ No 024 & 1932 & 1937 \\
\hline \multicolumn{4}{|c|}{ Five child families } \\
\hline & $M(S) 12 / 59 \mathrm{sb} ; \mathrm{F}(\mathrm{S}) 10 / 60 ; \mathrm{F9} / 63 ; \mathrm{M} 7 / 66 ;{ }^{*} \mathrm{M}(\mathrm{S}) 12 / 72$ & 1937 & 1934 \\
\hline & m- $/ 62 ; \mathrm{M}(\mathrm{A}) 10 / 63 \mathrm{sb} ; \mathrm{M} 11 / 64 ; \mathrm{M}(\mathrm{S}) 4 / 66$ No $069 ; \mathrm{F} 7 / 68 ; * \mathrm{~F}(\mathrm{~S}) 11 / 72$ & 1939 & 1939 \\
\hline & $\mathrm{M}(\mathrm{S}) 9 / 59 ; \mathrm{M}(\mathrm{S}) 12 / 60 ; \mathbf{F}(\mathrm{S}) 10 / 62 ; \mathrm{M} 1 / 65 ; \mathrm{M}(\mathrm{S}) 5 / 66$ No 089 & 1935 & 1934 \\
\hline \multicolumn{4}{|c|}{ Six child families } \\
\hline 064 & $\mathrm{M} 4 / 56 ; \mathrm{M} 11 / 57 ; \mathrm{F}(\mathrm{S}) 2 / 60 ; \mathrm{M} 4 / 63 ; \mathrm{F}(\mathrm{S}) 6 / 65$ No $074 ; \mathrm{M}(\mathrm{S}) 8 / 66$ & 1936 & 1939 \\
\hline Seven $+c l$ & I families & & \\
\hline & $\mathrm{M}(\mathrm{A}) 9 / 63 \mathrm{sb} ; \mathrm{F} 2 / 65 ; \mathrm{F} 5 / 67 ; \mathrm{M}(\mathrm{S}) 9 / 70$ No $043 ;{ }^{*} \mathrm{~F}(\mathrm{~S}) 2 / 73 ; \mathrm{F} 2 / 75 ; \mathrm{M} 10 / 77$ & 1936 & 1936 \\
\hline & $\mathrm{m} 10 / 63 ; \mathrm{M}(\mathrm{S}) 12 / 64 ; \mathrm{M} 1 / 66 ; \mathrm{m} 6 / 66 ; \mathrm{F} 8 / 67 ; \mathrm{F} 7 / 68 ; \mathrm{M}(\mathrm{S}) 4 / 70$ No $040 ;{ }^{*} \mathrm{M}(\mathrm{S}) 8 / 71 ; \mathrm{F}(\mathrm{S}) 1 / 74$ & 1940 & 1932 \\
\hline & $\mathrm{F} 2 / 56 ; \mathrm{F}(\mathrm{S}) 6 / 57 ; \mathrm{M} 1 / 59 ; \mathrm{M}(\mathrm{S}) 9 / 61 \mathrm{sb} ; \mathrm{F} 3 / 62 ; \mathrm{M} 7 / 65 ;{ }^{*} \mathrm{M}(\mathrm{S}) 10 / 66$ & 1940 & 1934 \\
\hline & $\mathrm{M} 1 / 50 ; \mathrm{F} 1 / 52 ; \mathrm{M} 11 / 52 ; \mathrm{F}(\mathrm{S}) 9 / 53 ; \mathrm{M} 12 / 54 ; \mathrm{M} 10 / 56 ; \mathrm{M}(\mathrm{S})-159 \mathrm{sb} ; \mathrm{F} / 60 ;[\mathrm{m}-/ 64 ; \mathrm{m}-/ 64] ; \mathrm{F}(\mathrm{S}) 4 / 66 ; \mathrm{F} 6 / 70$ & 1926 & 1928 \\
\hline & $\mathrm{M} 11 / 48 ; \mathrm{F} 1 / 51 ; \mathrm{M} 5 / 52 ; \mathrm{F}(\mathrm{S}) 9 / 53 ; \mathrm{m} 11 / 54 ; \mathrm{M} 12 / 55 ; \mathrm{M} 10 / 57 ; \mathrm{M}(\mathrm{S}) 2 / 60 ; \mathrm{m} 8 / 60 ; \mathrm{F} 2 / 62 ; \mathrm{m} 3 / 63 ; \mathrm{m6} / 64 ;$ & & \\
\hline & $\mathrm{m} 9 / 64 ; \mathrm{m} 3 / 65 ; \mathrm{F}(\mathrm{S}) 6 / 66 ; \mathrm{m}-167 ; \mathrm{m}-168 ; \mathrm{F} 6 / 70$ & 1926 & 1922 \\
\hline & $\mathrm{F} 10 / 54 ; \mathrm{F} 12 / 55 ; \mathrm{M} 2 / 56 \mathrm{sb} ; \mathrm{M} 1 / 58 ; \mathrm{F}(\mathrm{S}) 4 / 59 ; \mathrm{M} 8 / 60 ; \mathrm{m} 4 / 61 ; \mathrm{F}(\mathrm{S}) 2 / 62$ No $86 ;{ }^{*} \mathrm{M}(\mathrm{S}) 7 / 64 ; \mathrm{F} 10 / 69$ & 1935 & 1933 \\
\hline G. SPINA BI & DA (INDEX PATIENT): THREE AFFECTED OLDER SIBS & & \\
\hline $\begin{array}{l}\text { Four child } \\
024\end{array}$ & 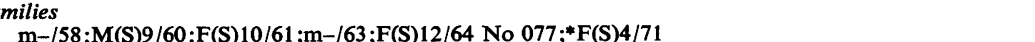 & 1932 & 1937 \\
\hline & 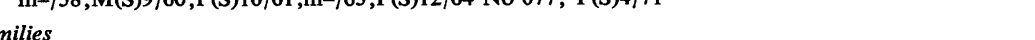 & & \\
\hline 089 & $\begin{array}{l}\text { milies } \\
\text { M(S)9/59;M(S) } 12 / 60 ; \mathrm{F}(\mathrm{S}) 10 / 62 \text { No } 088 ; \mathrm{M} 1 / 65 ; * \mathrm{M}(\mathrm{S}) 5 / 66\end{array}$ & 1935 & 1934 \\
\hline Seven $+c$ & Id families & & \\
\hline 082 & $\begin{array}{l}\mathrm{m}-/ 55 ; \mathrm{F}(\mathrm{S}) 1 / 56 \mathrm{sb} ; \mathrm{F}(\mathrm{S}) 9 / 56 \mathrm{sb} ; \mathrm{M} 10 / 57 ; \mathrm{m}-/ 58 ; \mathrm{M} 7 / 59 ; \mathrm{M}(\mathrm{S}) 9 / 60 ; \mathrm{m}-/ 61 ; \mathrm{F} 9 / 62 ;{ }^{*} \mathrm{~F}(\mathrm{~S}) 6 / 63 \mathrm{sb} \\
\mathrm{m}-/ 64 ; \mathrm{M} 2 / 66 ; \mathrm{M}(\mathrm{S}) 7 / 67 \text { No } 057 ; \mathrm{F} 12 / 68 ; \mathrm{F} 1 / 72\end{array}$ & 1937 & 1935 \\
\hline $\begin{array}{l}\text { H. SPINA BI } \\
\text { Seven }+c\end{array}$ & DA (INDEX PATIENT): FOUR AFFECTED OLDER SIBS & & \\
\hline 057 & m-155;F(S)1/56sb;F(S)9/56sb;M10/57;m-/58;M7/59;M(S)9/60;m-/61;F9/62;F(S)6/63sb & & \\
\hline & No $082 ; \mathrm{m}-/ 64 ; \mathrm{M} 2 / 66 ; \mathrm{M}(\mathrm{S}) 7 / 67 ; \mathrm{F} 12 / 68 ; \mathrm{F} 1 / 72$ & 1937 & 1935 \\
\hline
\end{tabular}

